\title{
State Estimation for Discrete-Time Fuzzy Cellular Neural Networks with Mixed Time Delays
}

\author{
Lijie Geng, ${ }^{1}$ Haiying $\mathrm{Li}^{2}{ }^{2}$ Bingchen Zhao, ${ }^{2}$ and Guang Su${ }^{2}$ \\ ${ }^{1}$ Institute of Applied Mathematics, Shijiazhuang Mechanical Engineering College, Shijiazhuang 050003, China \\ ${ }^{2}$ College of Mathematics and Information Technology, Xingtai University, Xingtai 054001, China \\ Correspondence should be addressed to Lijie Geng; genglijie1218@aliyun.com
}

Received 26 December 2013; Accepted 10 March 2014; Published 29 April 2014

Academic Editor: Qintao Gan

Copyright (c) 2014 Lijie Geng et al. This is an open access article distributed under the Creative Commons Attribution License, which permits unrestricted use, distribution, and reproduction in any medium, provided the original work is properly cited.

\begin{abstract}
This paper is concerned with the exponential state estimation problem for a class of discrete-time fuzzy cellular neural networks with mixed time delays. The main purpose is to estimate the neuron states through available output measurements such that the dynamics of the estimation error is globally exponentially stable. By constructing a novel Lyapunov-Krasovskii functional which contains a triple summation term, some sufficient conditions are derived to guarantee the existence of the state estimator. The linear matrix inequality approach is employed for the first time to deal with the fuzzy cellular neural networks in the discrete-time case. Compared with the present conditions in the form of $M$-matrix, the results obtained in this paper are less conservative and can be checked readily by the MATLAB toolbox. Finally, some numerical examples are given to demonstrate the effectiveness of the proposed results.
\end{abstract}

\section{Introduction}

Cellular neural networks (CNNs), initially proposed by Chua and Yang in 1988 [1], have been extensively investigated owing to their important applications in many areas such as image processing, pattern recognition, and combinatorial optimization. However, when mathematically modeling real neural networks, uncertainty or vagueness is often encountered. In order to take this vagueness into consideration, the fuzzy cellular neural networks (FCNNs) were proposed by Yang et al. in $[2,3]$, which integrate fuzzy logic into the structure of traditional CNNs and maintain local connectedness among cells. Recently, the dynamics analysis problem of FCNNs has received an increasing research attention and some relevant results have been reported in the literature [4-9].

It should be noted that all of the aforementioned results are in the continuous-time settings. In reality, however, discrete-time neural networks (DNNs) become more important than their continuous-time counterparts when implementing the neural networks in a digital way. Therefore, it is necessary to study the dynamics of DNNs and many results have been obtained during the past years [10-17]. By using the average dwell time approach and the discontinuous piecewise Lyapunov function technique, Zhang and Yu [16] studied passivity analysis problem for a class of discretetime switched neural networks with various activation functions and mixed time delays. More recently, Wu et al. [17] discussed the problem of dissipativity analysis for discretetime stochastic neural networks with time-varying discrete and finite-distributed delays with the aid of Jensen inequality and lower bounds lemma. Unfortunately, little attention has been paid to the discrete-time fuzzy cellular neural networks. In this context, only two works can be found $[18,19]$. The following discrete-time fuzzy cellular neural networks with variable delays and impulses were studied in [18]:

$$
\begin{aligned}
u_{i}(m+1)= & c_{i} u_{i}(m)+\sum_{j=1}^{n} a_{i j} f_{j}\left(u_{j}(m)\right) \\
& +\sum_{j=1}^{n} b_{i j} \vartheta_{j}+I_{i}+\bigwedge_{j=1}^{n} \alpha_{i j} f_{j}\left(u_{j}\left(m-\tau_{i j}(m)\right)\right)
\end{aligned}
$$




$$
\begin{aligned}
& +\bigvee_{j=1}^{n} \beta_{i j} f_{j}\left(u_{j}\left(m-\tau_{i j}(m)\right)\right) \\
& +\bigwedge_{j=1}^{n} T_{i j} \vartheta_{j}+\bigvee_{j=1}^{n} H_{i j} \vartheta_{j}, \quad m \neq m_{k},
\end{aligned}
$$

$$
\begin{aligned}
u_{i}(m)= & p_{i k}\left(u_{1}\left(m^{-}\right), \ldots, u_{n}\left(m^{-}\right)\right) \\
& +q_{i k}\left(u_{1}\left(\left(m-\tau_{i 1}(m)\right)^{-}\right), \ldots,\right. \\
& \left.u_{n}\left(\left(m-\tau_{i n}(m)\right)^{-}\right)\right)+J_{i k}, \quad m=m_{k},
\end{aligned}
$$

where $k=1,2, \ldots, i=1,2, \ldots, n, n$ corresponds to the number of units in the neural networks; $u_{i}(m)$ is the state of the $i$ th neuron at time $m ; f_{j}(\cdot)$ denotes the neuron activation function; $\tau_{i j}(m)$ corresponds to the transmission delay and satisfies $0<\tau_{i j}(m)<\tau ; T_{i j}, H_{i j}, \alpha_{i j}$, and $\beta_{i j}$ are elements of fuzzy feed-forward MIN template, fuzzy feed-forward MAX template, fuzzy feedback MIN template, and fuzzy feedback MAX template, respectively; $a_{i j}$ and $b_{i j}$ are elements of feedback template and feed-forward template, respectively; $\bigwedge$ and $\bigvee$ denote the fuzzy AND and fuzzy OR operation, respectively; $\vartheta_{j}$ and $I_{j}$ denote input and bias of the $i$ th neuron, respectively; $c_{i} \in(0,1)$. The time sequence $\left\{m_{k}\right\}$ satisfies $0 \leq m_{1}<m_{2}<\cdots,<m_{k}<\cdots$, and $\lim _{k \rightarrow+\infty}=+\infty$; $p_{i k}\left(u_{1}\left(m^{-}\right), \ldots, u_{n}\left(m^{-}\right)\right)$represents impulsive perturbation of the $i$ th unit at time $m_{k} ; q_{i k}\left(u_{1}\left(\left(m-\tau_{i 1}(m)\right)^{-}\right), \ldots, u_{n}((m-\right.$ $\left.\left.\tau_{\text {in }}(m)\right)^{-}\right)$) denotes impulsive perturbation of the $i$ th unit at time $m_{k}$ which is caused by the transmission delays; $J_{i k}$ represents external impulsive input at time $m_{k}$.

In [18], by using M-matrix theory and analytic methods, several sufficient conditions guaranteeing the global exponential stability of the equilibrium point and the existence of periodic solutions were obtained. Recently, Li and Wang [19] further discussed the existence and global exponential stability of equilibrium for discrete-time fuzzy BAM neural networks with variable delays and impulses. However, there still exist two points waiting for the improvements. First, in $[18,19]$, the authors only discussed discrete-time fuzzy neural networks with discrete delays. We all know the effects of distributed delays should not be neglected because a neural network usually has a spatial nature due to the presence of an amount of parallel pathways of a variety of node sizes and lengths. Second, the results in $[18,19]$ are described in the form of M-matrix. However, the results in the form of Mmatrix do not contain any unknown parameters. Moreover, the excitatory and inhibitory effects of neuron on neural networks are also neglected. Thus, the conservativeness of the results is much greater. We note that the results in the form of linear matrix inequalities (LMIs) are less conservative because they not only include suitable number of unknown parameters, but also consider the excitatory and inhibitory effects of neuron on neural networks. Furthermore, the LMIs can be easily solved by using the MATLAB LMI toolbox.

In many applications, one needs to know the neuron states to achieve certain objectives. On the other hand, the neuron states are not often fully available in the network outputs. Thus, the state estimation problem for neural networks has drawn particular research interests [20-24]. The results reported in [20-24] can only ensure that the dynamics of the error system is asymptotically stable. In some cases, the engineers need to know how fast the trajectory of the error system converges to the equilibrium point. Therefore, the problem of exponential state estimation is very important. Reference [25] investigated the problem of exponential state estimation for Markovian jumping neural networks with time-varying discrete and distributed delays. By constructing a novel Lyapunov-Krasovskii functional and developing a new convex combination technique, a new exponential stability condition was proposed. Reference [26] studied the estimator design problem for discrete-time switched neural networks with time-varying delay and addressed the asynchronous phenomenon between the neuron state-mode switching and the estimator switching. Delay-dependent sufficient conditions were provided to ensure the exponential stability of estimation error dynamics as well as a prescribed $l_{2}$ gain level from the noise signal to the estimation error. Recently, the authors in [27] discussed the state estimation problem for continuous-time fuzzy cellular neural networks. However, to the best of our knowledge, the state estimation problem for discrete-time fuzzy cellular neural networks has not been investigated in the existing literatures, which elicits our research work.

In this paper, the state estimation problem for a class of discrete-time fuzzy cellular neural networks is considered for the first time. The neural networks under study involve fuzzy parameters, discrete delays, and unbounded distributed delays, which are more general than those discussed in the previous literatures. By constructing a Lyapunov-Krasovskii functional including the triple-integral term, some delaydependent sufficient conditions are derived, such that, for all admissible delay bounds, the dynamics of the estimation error is globally exponentially stable. It is noted that the effects of neuron excitatory and inhibitory responses on neural networks are taken into account in the proposed approach, which will lead to less conservative results. These conditions obtained are in the form of LMIs whose solution can be easily calculated by using MATLAB LMI toolbox.

\section{Problem Formulation}

Considering the following discrete-time fuzzy cellular neural networks with mixed time delays:

$$
\begin{aligned}
x_{i}(k+1)= & -d_{i} x_{i}(k)+\sum_{j=1}^{n} a_{i j} g_{j}\left(x_{j}(k)\right) \\
& +\sum_{j=1}^{n} b_{i j} g_{j}\left(x_{j}(k-\tau(k))\right)+\sum_{j=1}^{n} v_{i j} u_{j}+I_{i} \\
& +\bigwedge_{j=1}^{n} \alpha_{i j} \sum_{m=1}^{+\infty} \mu_{m} g_{j}\left(x_{j}(k-m)\right)
\end{aligned}
$$




$$
\begin{aligned}
& +\bigvee_{j=1}^{n} \beta_{i j} \sum_{m=1}^{+\infty} \mu_{m} g_{j}\left(x_{j}(k-m)\right) \\
& +\bigwedge_{j=1}^{n} \gamma_{i j} u_{j}+\bigvee_{j=1}^{n} \chi_{i j} u_{j}
\end{aligned}
$$

where $i=1,2, \ldots, n, n$ is the number of neurons in the networks; $x_{i}(k)$ is the state of the $i$ th neuron at time $k ; \alpha_{i j}, \beta_{i j}, \gamma_{i j}$, and $\chi_{i j}$ are elements of fuzzy feedback MIN template, fuzzy feedback MAX template, fuzzy feed-forward MIN template, and fuzzy feed-forward MAX template, respectively; $a_{i j}$ and $b_{i j}$ are elements of feedback template and $v_{i j}$ are elements of feed-forward template; $\bigwedge$ and $\bigvee$ denote the fuzzy AND and fuzzy OR operation, respectively; $u_{i}$ and $I_{i}$ denote input and bias of the $i$ th neuron, respectively. $f_{j}(\cdot)$ denotes the neuron activation function; $\tau(k)$ denotes the time-varying delay and satisfies $\tau_{m} \leq \tau(k) \leq \tau_{M}$, in which $\tau_{m}$ and $\tau_{M}$ are known positive integers; $d_{i}$ satisfies $0<d_{i}<1$ and represents the rate with which the $i$ th neuron will reset its potential to the resting state when disconnected from the network and external inputs.

Throughout the paper, the following assumptions are needed.

(H1) The constant $\mu_{m} \geq 0(m=1,2, \ldots)$ satisfies the following convergent conditions:

$$
\begin{gathered}
\sum_{m=1}^{+\infty} \mu_{m}=\bar{\mu}<+\infty, \quad \sum_{m=1}^{+\infty} m \mu_{m}=\widehat{\mu}<+\infty, \\
\sum_{m=1}^{+\infty} m \mu_{m} \theta^{m}=w(\theta)<+\infty, \\
\theta>1 .
\end{gathered}
$$

(H2) For $i \in\{1,2, \ldots, n\}$, the neuron activation function $g_{i}(\cdot)$ satisfies

$$
l_{i}^{-} \leq \frac{g_{i}\left(\zeta_{1}\right)-g_{i}\left(\zeta_{2}\right)}{\zeta_{1}-\zeta_{2}} \leq l_{i}^{+}, \quad \forall \zeta_{1}, \zeta_{2} \in R\left(\zeta_{1} \neq \zeta_{2}\right)
$$

where $l_{i}^{-}, l_{i}^{+}$are some constants.

Remark 1. This assumption was first introduced in [28]. The constants $l_{i}^{-}, l_{i}^{+}$are allowed to be positive, negative, or zero. Hence, the resulting activation functions could be nonmonotonic and more general than the usual sigmoid functions and Lipschitz-type conditions. Such a description is very precise in quantifying the lower and upper bounds of the activation functions and therefore very helpful for employing LMI-based method to reduce the possible conservatism.

As mentioned before, for relatively large scale neural networks, it is very difficult to acquire the complete information of the neuron states. The purpose of this study is to develop an efficient approach to estimate the states of neural networks via the available network outputs. Here, we assume the network outputs to be of the form

$$
y(k)=C x(k)+\varphi(k, x(k)),
$$

where $y(k) \in \mathbb{R}^{m}, C$ is a known constant matrix with appropriate dimension, and $\varphi: \mathbb{R} \times \mathbb{R}^{n} \rightarrow \mathbb{R}^{m}$ is the nonlinear disturbance satisfying the following Lipschitz condition:

$$
|\varphi(k, x)-\varphi(k, y)| \leq|H(x-y)|, \quad \forall k \in \mathbb{N}, x, y \in \mathbb{R}^{n},
$$

where $H \in \mathbb{R}^{m \times n}$ is also a known constant matrix.

We construct the state estimator as follows:

$$
\begin{aligned}
\widehat{x}_{i}(k+1)= & -d_{i} \widehat{x}_{i}(k)+\sum_{j=1}^{n} a_{i j} g_{j}\left(\widehat{x}_{j}(k)\right) \\
& +\sum_{j=1}^{n} b_{i j} g_{j}\left(\widehat{x}_{j}(k-\tau(k))\right)+\sum_{j=1}^{n} v_{i j} u_{j}+I_{i} \\
& +\bigwedge_{j=1}^{n} \alpha_{i j} \sum_{m=1}^{+\infty} \mu_{m} g_{j}\left(\hat{x}_{j}(k-m)\right) \\
& +\bigvee_{j=1}^{n} \beta_{i j} \sum_{m=1}^{+\infty} \mu_{m} g_{j}\left(\hat{x}_{j}(k-m)\right) \\
& +\bigwedge_{j=1}^{n} \gamma_{i j} u_{j}+\bigvee_{j=1}^{n} \chi_{i j} u_{j} \\
& +\sum_{l=1}^{m} k_{i l}\left[y_{l}(k)-\sum_{j=1}^{n} c_{l j} \hat{x}_{j}(k)-\varphi_{l}\left(k, \widehat{x}_{j}(k)\right)\right],
\end{aligned}
$$

where $\widehat{x}_{i}$ is the estimation of the $i$ th neuron state and $k_{i l}$ is the element of an estimator gain matrix to be designed.

Define the error $e_{i}(k)=x_{i}(k)-\widehat{x}_{i}(k)$. Then the error system can be directly obtained from (3) and (8):

$$
\begin{aligned}
e_{i}(k+1)= & -d_{i} e_{i}(k)-\sum_{l=1}^{m} k_{i l} \sum_{j=1}^{n} c_{l j} e_{j}(k) \\
& +\sum_{j=1}^{n} a_{i j} f_{j}(k)+\sum_{j=1}^{n} b_{i j} f_{j}(k-\tau(k)) \\
& +\bigwedge_{j=1}^{n} \alpha_{i j} \sum_{m=1}^{+\infty} \mu_{m} g_{j}\left(x_{j}(k-m)\right) \\
& -\bigwedge_{j=1}^{n} \alpha_{i j} \sum_{m=1}^{\infty} \mu_{m} g_{j}\left(\hat{x}_{j}(k-m)\right) \\
& +\bigvee_{j=1}^{n} \beta_{i j} \sum_{m=1}^{+\infty} \mu_{m} g_{j}\left(x_{j}(k-m)\right) \\
& -\bigvee_{j=1}^{n} \beta_{i j} \sum_{m=1}^{+\infty} \mu_{m} g_{j}\left(\hat{x}_{j}(k-m)\right)-\sum_{l=1}^{m} k_{i l} h_{l}(k),
\end{aligned}
$$


where $f_{j}(k)=g_{j}\left(x_{j}(k)\right)-g_{j}\left(\hat{x}_{j}(k)\right)$ and $h_{l}(k)=\varphi_{l}\left(k, x_{j}(k)\right)-$ $\varphi_{l}\left(k, \widehat{x}_{j}(k)\right)$. The initial condition associated with (9) is given as $e(s)=\psi(s)(s=0,-1,-2, \ldots)$. It is obvious that $e(k, 0) \equiv 0$ is a trivial solution of the system (9).

Now we introduce the following definition and lemmas which will be used in the sequel.

Lemma 2 (see [2]). Let $x$ and $y$ be two states of system (3), then one has

$$
\begin{gathered}
\left|\bigwedge_{j=1}^{n} \alpha_{i j} h_{j}\left(x_{j}(s)\right)-\bigwedge_{j=1}^{n} \alpha_{i j} h_{j}\left(y_{j}(s)\right)\right| \\
\leq \sum_{j=1}^{n}\left|\alpha_{i j}\right|\left|h_{j}\left(x_{j}(j)\right)-h_{j}\left(y_{j}(j)\right)\right|, \\
\left|\bigvee_{j=1}^{n} \beta_{i j} h_{j}\left(x_{j}(s)\right)-\bigvee_{j=1}^{n} \beta_{i j} h_{j}\left(y_{j}(s)\right)\right| \\
\leq \sum_{j=1}^{n}\left|\beta_{i j}\right|\left|h_{j}\left(x_{j}(j)\right)-h_{j}\left(y_{j}(j)\right)\right| .
\end{gathered}
$$

Lemma 3 (see [7]). For any constant $\epsilon>0$, n-dimensional real vectors $x, y$, and positive definite matrix $N \in \mathbb{R}^{n \times n}$, the following matrix inequality holds:

$$
2 x^{T} y \leq \epsilon x^{T} N x+\epsilon^{-1} y^{T} N^{-1} y .
$$

Lemma 4 (see $[29,30]$ ). Let $M \in \mathbb{R}^{n \times n}$ be a positive semidefinite matrix, let $x_{i} \in \mathbb{N}^{n}$ be a vector, and let $a_{i} \geq 0$ $(i=1,2, \ldots)$ be scalar. If the series concerned are convergent, then the following inequality holds:

$$
\begin{gathered}
(1)\left(\sum_{m=1}^{+\infty} a_{i} x_{i}\right)^{T} M\left(\sum_{m=1}^{+\infty} a_{i} x_{i}\right) \leq\left(\sum_{m=1}^{+\infty} a_{i}\right) \sum_{m=1}^{+\infty} a_{i} x_{i}^{T} M x_{i}, \\
\text { (2) }\left[\sum_{i=-m}^{i=-n-1} \sum_{j=i}^{-1} x_{j}\right]^{T} M\left[\sum_{i=-m}^{i=-n-1} \sum_{j=i}^{-1} x_{j}\right] \\
\leq \frac{m-n}{2}(m+n+1) \sum_{i=-m}^{i=-n-1} \sum_{j=i}^{-1} x_{j}^{T} M x_{j} .
\end{gathered}
$$

Definition 5. The error system (9) is said to be globally exponentially stable, if there exist constants $\alpha>0$ and $0<$ $\mu<1$ such that

$$
\|e(k)\| \leq \alpha \mu^{k} \max _{-\infty<s \leq 0}\|e(s)\|, \quad \forall k \geq 0
$$

where $\|e(k)\|$ is the Euclidean norm of $e(k)$.

\section{Main Results}

In this section, by constructing a new Lyapunov-Krasovskii functional, we will develop an LMI approach to derive some sufficient conditions under which the error system (9) is globally exponentially stable, and the resulting gain matrix $\mathrm{K}$ will also be given. For presentation convenience, in the following, we denote

$$
\begin{gathered}
L_{1}=\operatorname{diag}\left(l_{1}^{-} l_{1}^{+}, l_{2}^{-} l_{2}^{+}, \ldots, l_{n}^{-} l_{n}^{+}\right), \\
L_{2}=\operatorname{diag}\left(\frac{l_{1}^{-}+l_{1}^{+}}{2}, \frac{l_{2}^{-}+l_{2}^{+}}{2}, \ldots, \frac{l_{n}^{-}+l_{n}^{+}}{2}\right), \\
\tau_{1}=\tau_{M}-\tau_{m}, \quad \tau_{2}=\tau_{M}+\tau_{m} .
\end{gathered}
$$

Theorem 6. Suppose that assumptions (H1)-(H2) and condition (7) hold; the error system (9) is globally exponentially stable if there exist positive diagonal matrices $P, D_{1}$, and $D_{2}$, positive symmetric matrices $Q_{1}, Q_{2}, T, S$, and $R$, real matrix $N$, and scalars $\epsilon>0, \vartheta>0$ such that the following $L M I$ holds:

$$
\Phi=\left[\begin{array}{cc}
\Psi & \xi^{T} \\
* & -\epsilon n^{-1} I
\end{array}\right]<0,
$$

where

$$
\Psi=\left[\begin{array}{ccccccccccc}
r_{1,1} & 0 & 0 & 0 & r_{1,5} & r_{1,6} & r_{1,7} & r_{1,8} & r_{1,9} & 0 & -N \\
* & -Q_{1} & 0 & 0 & 0 & 0 & 0 & 0 & 0 & 0 & 0 \\
* & * & -Q_{2} & 0 & 0 & 0 & 0 & 0 & 0 & 0 & 0 \\
* & * & * & r_{4,4} & 0 & 0 & 0 & 0 & L_{2} D_{2} & 0 & 0 \\
* & * & * & * & r_{5,5} & 0 & 0 & P A & P B & 0 & -N \\
* & * & * & * & * & r_{6,6} & r_{6,7} & 0 & 0 & 0 & 0 \\
* & * & * & * & * & * & r_{7,7} & 0 & 0 & 0 & 0 \\
* & * & * & * & * & * & * & r_{8,8} & 0 & 0 & 0 \\
* & * & * & * & * & * & * & * & -D_{2} & 0 & 0 \\
* & * & * & * & * & * & * & * & * & r_{10,10} & 0 \\
* & * & * & * & * & * & * & * & * & * & -9 I
\end{array}\right],
$$


with

$$
\begin{gathered}
r_{1,1}=-2 P D-N C-C^{T} N^{T}-P+Q_{1}+Q_{2} \\
+\left(\tau_{1}+1\right) T-\frac{2 \tau_{1}}{\tau_{2}+1} R-L_{1} D_{1}+9 H^{T} H, \\
r_{1,5}=-D^{T} P-C^{T} N^{T}-P, \quad r_{1,6}=r_{1,7}=\frac{2}{\tau_{2}+1} R, \\
r_{1,8}=P A+L_{2} D_{1}, \quad r_{1,9}=P B, \\
r_{4,4}=-T-L_{1} D_{2}, \quad r_{5,5}=-P+\frac{\tau_{1}}{2}\left(\tau_{2}+1\right) R, \\
r_{6,6}=r_{6,7}=r_{7,7}=-\frac{2}{\tau_{1}\left(\tau_{2}+1\right)} R, \\
r_{8,8} S-D_{1}, \quad r_{10,10}=n Y^{T} P Y+\epsilon I-\frac{1}{\bar{\mu}} S, \\
|\alpha|_{r}=\operatorname{diag}\left\{\sum_{i=1}^{n}\left|\alpha_{i 1}\right|, \sum_{i=1}^{n}\left|\alpha_{i 2}\right|, \ldots, \sum_{i=1}^{n}\left|\alpha_{i n}\right|\right\} \\
|\beta|_{r}=\operatorname{diag}\left\{\sum_{i=1}^{n}\left|\beta_{i 1}\right|, \sum_{i=1}^{n}\left|, \beta_{i 2}\right|, \ldots, \sum_{i=1}^{n}\left|\beta_{i n}\right|\right\}
\end{gathered}
$$

Moreover, the estimate gain matrix can be designed as $K=$ $P^{-1} N$.

Proof. Defining $\eta(k)=e(k+1)-e(k)$, we introduce the following Lyapunov-Krasovskii functional:

$$
V(k)=\sum_{i=1}^{5} V(k),
$$

where

$$
\begin{aligned}
& V_{1}(k)=e^{T}(k) P e(k), \\
& V_{2}(k)=\sum_{i=k-\tau_{m}}^{k-1} e^{T}(i) Q_{1} e(i)+\sum_{i=k-\tau_{M}}^{k-1} e^{T}(i) Q_{2} e(i), \\
& V_{3}(k)=\sum_{i=-\tau_{M}+1} \sum_{j=k-1+i}^{k-\tau_{m}+1} e(j)^{T} T e(j), \\
& V_{4}(k)=\sum_{i=1}^{+\infty} \mu_{i} \sum_{j=k-i}^{k-1} f^{T}(j) S f(j), \\
& V_{5}(k)=\sum_{i=-\tau_{M}} \sum_{j=i}^{-\tau_{m}-1} \sum_{l=k+j}^{k-1} \eta^{T}(l) R \eta(l) .
\end{aligned}
$$

According to Lemma 2, the following inequalities hold:

$$
\begin{aligned}
& \left|\bigwedge_{j=1}^{n} \alpha_{i j} \sum_{m=1}^{+\infty} \mu_{m} g_{j}\left(x_{j}(k)\right)-\bigwedge_{j=1}^{n} \alpha_{i j} \sum_{m=1}^{\infty} \mu_{m} g_{j}\left(\hat{x}_{j}(k)\right)\right| \\
& \quad \leq \sum_{j=1}^{n}\left|\alpha_{i j}\right|\left|\sum_{m=1}^{+\infty} \mu_{m} f_{j}(k)\right|, \\
& \left|\bigwedge_{j=1}^{n} \beta_{i j} \sum_{m=1}^{+\infty} \mu_{m} g_{j}\left(x_{j}(k)\right)-\bigwedge_{j=1}^{n} \beta_{i j} \sum_{m=1}^{\infty} \mu_{m} g_{j}\left(\hat{x}_{j}(k)\right)\right| \\
& \leq \sum_{j=1}^{n}\left|\beta_{i j}\right|\left|\sum_{m=1}^{+\infty} \mu_{m} f_{j}(k)\right| .
\end{aligned}
$$

Calculating the difference of $V(k)$ along the solution of system (9), we have

$$
\Delta V(k)=\sum_{i=1}^{5} \Delta V(k),
$$

where

$$
\begin{aligned}
\Delta V_{1}(k)= & V_{1}(k+1)-V_{1}(k) \\
= & \eta^{T}(k) P \eta(k)+2 e^{T}(k) P \eta(k) \\
= & \eta^{T}(k) P \eta(k) \\
+2 \sum_{i=1}^{n} p_{i} e_{i}(k)\left[\begin{array}{l}
- \\
\quad
\end{array}\right. & \left.-\sum_{i}+1\right) e_{i}(k) \\
& +\sum_{j=1}^{m} k_{i l} \sum_{j=1}^{n} c_{i j} f_{j} e_{j}(k)+\sum_{j=1}^{n} a_{i j} f_{j}(k) \\
& +\bigwedge_{j=1}^{n} \alpha_{i j} \sum_{m=1}^{+\infty} \mu_{m} g_{j}\left(x_{j}(k-m)\right) \\
& -\bigvee_{j=1}^{n} \beta_{i j} \sum_{m=1}^{+\infty} \mu_{m} g_{j}\left(\hat{x}_{j}(k-m)\right) \\
& \left.+\bigvee_{j=1}^{+\infty} \alpha_{i j} \sum_{m=1}^{n} \mu_{m} g_{j} \sum_{j=1}^{+\infty} \hat{x}_{j}(k-m)\right)
\end{aligned}
$$




$$
\begin{aligned}
& \leq \eta^{T}(k) P \eta(k)+2 e^{T}(k) \\
& \times P[-(D+K C+I) e(k)+A f(k) \\
& +B f(k-\tau(k))-K h(k)] \\
& +2|e(k)|^{T} P(|\alpha|+|\beta|)\left|\sum_{m=1}^{+\infty} \mu_{m} f(k-m)\right| \\
& \leq \eta^{T}(k) P \eta(k)+2 e^{T}(k) \\
& \times P[-(D+K C+I) e(k)+A f(k) \\
& +B f(k-\tau(k))-K h(k)] \\
& +2|e(k)|^{T} P(|\alpha|+|\beta|)\left|\sum_{m=1}^{+\infty} \mu_{m} f(k-m)\right| \\
& +2 \eta^{T}(k) P[-\eta(k)+\eta(k)] \\
& \leq-\eta^{T}(k) P \eta(k)+2 e^{T}(k) \\
& \times P[-(D+K C+I) e(k)+A f(k) \\
& +B f(k-\tau(k))-K h(k)] \\
& +2|e(k)|^{T} P(|\alpha|+|\beta|)\left|\sum_{m=1}^{+\infty} \mu_{m} f(k-m)\right| \\
& +2 \eta^{T}(k) P[-(D+K C+I) e(k)+A f(k) \\
& +B f(k-\tau(k))-K h(k)] \\
& +2 \eta^{T}(k) P(|\alpha|+|\beta|)\left|\sum_{m=1}^{+\infty} \mu_{m} f(k-m)\right| \\
& \leq-\eta^{T}(k) P \eta(k)+2\left[e^{T}(k)+\eta^{T}(k)\right] \\
& \times P[-(D+K C+I) e(k)+A f(k) \\
& +B f(k-\tau(k))-K h(k)]+e(k)^{T} P e(k) \\
& +n \sum_{m=1}^{+\infty} \mu_{m} f^{T}(k-m)\left(|\alpha|_{r}+|\beta|_{r}\right)^{T} P\left(|\alpha|_{r}+|\beta|_{r}\right) \\
& \times \sum_{m=1}^{+\infty} \mu_{m} f(k-m)+\epsilon^{-1} n \eta^{T}(k) \\
& \times P\left(|\alpha|_{r}+|\beta|_{r}\right)\left(|\alpha|_{r}+|\beta|_{r}\right)^{T} P^{T} \eta(k) \\
& +\epsilon\left(\sum_{m=1}^{+\infty} \mu_{m} f^{T}(k-m)\right)\left(\sum_{m=1}^{+\infty} \mu_{m} f(k-m)\right),
\end{aligned}
$$

$$
\begin{aligned}
\Delta V_{2}(k)= & V_{2}(k+1)-V_{2}(k) \\
= & e^{T}(k)\left(Q_{1}+Q_{2}\right) e(k)-e^{T}\left(k-\tau_{m}\right) Q_{1} e\left(k-\tau_{m}\right) \\
& -e^{T}\left(k-\tau_{M}\right) Q_{2} e\left(k-\tau_{M}\right)
\end{aligned}
$$

$\Delta V_{3}(k)=V_{3}(k+1)-V_{3}(k)$

$=\sum_{i=-\tau_{M}+1}^{-\tau_{m}+1} \sum_{j=k+i}^{k} e(j)^{T} T e(j)-\sum_{i=-\tau_{M}+1}^{-\tau_{m}+1} \sum_{j=k-1+i}^{k-1} e(j)^{T} T e(j)$

$=\sum_{i=-\tau_{M}+1}^{-\tau_{m}+1}\left[e^{T}(k) T e(k)-e^{T}(k-1+i) T e(k-1+i)\right]$

$=\left(\tau_{1}+1\right) e^{T}(k) T e(k)-\sum_{i=k-\tau_{M}}^{k-\tau_{m}} e^{T}(i) T e(i)$

$\leq\left(\tau_{1}+1\right) e^{T}(k) T e(k)$

$$
-e^{T}(k-\tau(k)) T e(k-\tau(k)),
$$

$\Delta V_{4}(k)=V_{4}(k+1)-V_{4}(k)$

$=\sum_{i=1}^{+\infty} \mu_{i}\left[f^{T}(k) S f(k)-f^{T}(k-i) S f(k-i)\right]$

(by Lemma 4)

$$
\begin{aligned}
& \leq \bar{\mu} f^{T}(k) S f(k)-\frac{1}{\bar{\mu}}\left(\sum_{i=1}^{+\infty} \mu_{i} f(k-i)\right)^{T} \\
& \quad \times S\left(\sum_{i=1}^{+\infty} \mu_{i} f(k-i)\right)
\end{aligned}
$$

$\Delta V_{5}(k)=V_{5}(k+1)-V_{5}(k)$

$=\frac{\tau_{1}}{2}\left(\tau_{2}+1\right) \eta^{T}(k) R \eta(k)$

$-\sum_{i=-\tau_{M}}^{-\tau_{m}-1} \sum_{j=k+i}^{k-1} \eta^{T}(j) R \eta(j)$ (by Lemma 4)

$\leq \frac{\tau_{1}}{2}\left(\tau_{2}+1\right) \eta^{T}(k) R \eta(k)-\frac{2}{\tau_{1}\left(\tau_{2}+1\right)}$

$$
\times\left[\sum_{i=-\tau_{M}}^{-\tau_{m}-1} \sum_{j=k+i}^{k-1} \eta(j)\right]^{T} R\left[\sum_{i=-\tau_{M}}^{-\tau_{m}-1} \sum_{j=k+i}^{k-1} \eta(j)\right]
$$$$
\leq \frac{\tau_{1}}{2}\left(\tau_{2}+1\right) \eta^{T}(k) R \eta(k)-\frac{2}{\tau_{1}\left(\tau_{2}+1\right)}
$$$$
\times\left[\tau_{1} e(k)-\sum_{i=k-\tau(k)}^{k-\tau_{m}-1} e(i)-\sum_{i=k-\tau_{M}}^{k-\tau(k)-1} e(i)\right]^{T}
$$ 


$$
\begin{aligned}
& \times R\left[\tau_{1} e(k)-\sum_{i=k-\tau(k)}^{k-\tau_{m}-1} e(i)-\sum_{i=k-\tau_{M}}^{k-\tau(k)-1} e(i)\right] \\
& =\frac{\tau_{1}}{2}\left(\tau_{2}+1\right) \eta^{T}(k) R \eta(k) \\
& -\frac{2 \tau_{1}}{\tau_{2}+1} e^{T}(k) \operatorname{Re}(k) \\
& +\frac{4}{\tau_{2}+1}\left[e^{T}(k) R \sum_{i=k-\tau(k)}^{k-\tau_{m}-1} e(i)\right. \\
& \left.+e^{T}(k) R \sum_{i=k-\tau_{M}}^{k-\tau(k)-1} e(i)\right] \\
& -\frac{4}{\tau_{1}\left(\tau_{2}+1\right)}\left[\left(\sum_{i=k-\tau(k)}^{k-\tau_{m}-1} e(i)\right)^{T}\right. \\
& \left.\times R\left(\sum_{i=k-\tau_{M}}^{k-\tau(k)-1} e(i)\right)\right] \\
& -\frac{2}{\tau_{1}\left(\tau_{2}+1\right)}\left[\left(\sum_{i=k-\tau(k)}^{k-\tau_{m}-1} e(i)\right)^{T} R\left(\sum_{i=k-\tau(k)}^{k-\tau_{m}-1} e(i)\right)\right. \\
& +\left(\sum_{i=k-\tau_{M}}^{k-\tau(k)-1} e(i)\right)^{T} . \\
& \left.\times R\left(\sum_{i=k-\tau_{M}}^{k-\tau(k)-1} e(i)\right)\right]
\end{aligned}
$$

Moreover, from (5) and (7) we know that, for any $n$ dimensional diagonal matrices $L_{1}>0, L_{2}>0$ and any scalar $\vartheta>0$, the following inequalities hold:

$$
\begin{gathered}
{\left[\begin{array}{c}
e(k) \\
f(k)
\end{array}\right]^{T}\left[\begin{array}{cc}
L_{1} D_{1} & -L_{2} D_{1} \\
* & D_{1}
\end{array}\right]\left[\begin{array}{l}
e(k) \\
f(k)
\end{array}\right] \leq 0} \\
{\left[\begin{array}{c}
e(k-\tau(k)) \\
f(k-\tau(k))
\end{array}\right]^{T}\left[\begin{array}{cc}
L_{1} D_{2} & -L_{2} D_{2} \\
* & D_{2}
\end{array}\right]\left[\begin{array}{l}
e(k-\tau(k)) \\
f(k-\tau(k))
\end{array}\right] \leq 0} \\
\vartheta h^{T}(k) h(k) \leq \vartheta e^{T}(k) H^{T} H e(k) .
\end{gathered}
$$

Substituting (22)-(29) into (21) leads to

$$
\Delta V(k) \leq \delta^{T}(k)\left[\Psi+\xi^{T} \epsilon^{-1} n \xi\right] \delta(k),
$$

where

$$
\begin{gathered}
\delta^{T}(k)=\left[e^{T}(k), e^{T}\left(k-\tau_{m}\right), e^{T}\left(k-\tau_{M}\right), e^{T}(k-\tau(k)),\right. \\
\eta^{T}(k), \sum_{i=k-\tau(k)}^{k-\tau_{m}-1} e^{T}(i), \sum_{i=k-\tau_{M}}^{k-\tau(k)-1} e^{T}(i), f^{T}(k), \\
\left.f^{T}(k-\tau(k)), \sum_{i=1}^{+\infty} \mu_{i} f^{T}(k-i), h^{T}(k)\right] \\
\xi=\left[0,0,0,0,(P Y)^{T}, 0,0,0,0,0,0,0\right] .
\end{gathered}
$$

By Schur complement, (15) implies that there exists a constant $\varepsilon<0$ such that

$$
\Delta V(k) \leq \delta^{T}(k) \Omega \delta(k) \leq \varepsilon\|e(k)\|^{2},
$$

where

$$
\Omega=\Psi+\xi^{T} \epsilon^{-1} n \xi .
$$

By the definition of $V(k)$, it is easy to obtain

$$
\begin{aligned}
V(k) \leq & \rho_{1}\|e(k)\|^{2}+\rho_{2} \sum_{i=k-\tau_{M}}^{k-1}\|e(i)\|^{2}+\rho_{3} \sum_{i=k-\tau_{M}}^{k-1}\|e(i+1)\|^{2} \\
& +\rho_{4} \sum_{i=1}^{+\infty} \mu_{i} \sum_{j=k-i}^{k-1}\|e(j)\|^{2},
\end{aligned}
$$

where

$$
\begin{aligned}
\rho_{1}= & \lambda_{\max }(P), \\
\rho_{2}= & \lambda_{\max }\left(Q_{1}\right)+\lambda_{\max }\left(Q_{2}\right)+\left(\tau_{1}+1\right) \lambda_{\max }(T) \\
& +\tau_{1}\left(\tau_{2}+1\right) \lambda_{\max }(R), \\
\rho_{3}= & \tau_{1}\left(\tau_{2}+1\right) \lambda_{\max }(R), \\
\rho_{4}= & \lambda_{\max }\left(L^{T} S L\right), \\
L= & \operatorname{diag}\left\{l_{1}^{+}, l_{2}^{+}, \ldots, l_{n}^{+}\right\} .
\end{aligned}
$$

For any scalar $\theta>1$, it follows from (32) and (34) that

$$
\begin{array}{rl}
\theta^{k+1} & V(k+1)-\theta^{k} V(k) \\
= & \theta^{k+1} \Delta V(k)-\theta^{k}(\theta-1) V(k) \\
\leq & {\left[\theta^{k}(\theta-1) \rho_{1}-\theta^{k+1} \varepsilon\right]\|e(k)\|^{2}+\theta^{k}(\theta-1)} \\
& \times\left[\rho_{2} \sum_{i=k-\tau_{M}}^{k-1}\|e(i)\|^{2}+\rho_{3} \sum_{i=k-\tau_{M}}^{k-1}\|e(i+1)\|^{2}\right. \\
& \left.+\rho_{4} \sum_{i=1}^{+\infty} \mu_{i} \sum_{j=k-i}^{k-1}\|e(j)\|^{2}\right] .
\end{array}
$$


For any integer $N \geq \tau_{M}+1$, summing up both sides of (36) from 0 to $N-1$ with respect to $k$, we can obtain

$$
\begin{aligned}
\theta^{N} V(N) \leq V(0)+\left[(\theta-1) \rho_{1}-\theta \varepsilon\right] \sum_{k=0}^{N-1} \theta^{k}\|e(k)\|^{2} \\
+(\theta-1)\left[\rho_{2} \sum_{k=0}^{N-1} \sum_{i=k-\tau_{M}}^{k-1} \theta^{k}\|e(i)\|^{2}\right. \\
+\rho_{3} \sum_{k=0}^{N-1} \sum_{i=k-\tau_{M}}^{k-1} \theta^{k}\|e(i+1)\|^{2} \\
\left.+\rho_{4} \sum_{i=1}^{+\infty} \mu_{i} \sum_{k=0}^{N-1} \sum_{j=k-i}^{k-1} \theta^{k}\|e(j)\|^{2}\right] .
\end{aligned}
$$

By the methods employed in [31], we have

$$
\begin{aligned}
& \sum_{k=0}^{N-1} \sum_{i=k-\tau_{M}}^{k-1} \theta^{k}\|e(i)\|^{2} \leq \tau_{M} \theta^{\tau_{M}} \sup _{-\tau_{M} \leq s \leq 0}\|e(s)\|^{2} \\
& +\tau_{M} \theta^{\tau_{M}} \sum_{i=0}^{N-1} \theta^{i}\|e(i)\|^{2}
\end{aligned}
$$

$$
+i \theta^{i} \sum_{j=0}^{N-1} \theta^{j}\|e(j)\|^{2}
$$

In (34), let $k=0$ and from ( $\mathrm{H} 1)$ we can obtain

$$
\begin{aligned}
V(0) \leq & \rho_{1}\|e(0)\|^{2}+\rho_{2} \sum_{i=k-\tau_{M}}^{k-1}\|e(i)\|^{2} \\
& +\rho_{3} \sum_{i=k-\tau_{M}}^{k-1}\|e(i+1)\|^{2}+\rho_{4} \sum_{i=1}^{+\infty} \mu_{i} \sum_{j=k-i}^{k-1}\|e(j)\|^{2} \\
\leq & {\left[\rho_{1}+\tau_{M}\left(\rho_{2}+\rho_{3}\right)\right] \sup _{-\tau_{M} \leq s \leq 0}\|e(s)\|^{2} } \\
& +\rho_{4} \widehat{\mu} \sup _{-\tau_{M} \leq s \leq 0}\|e(s)\|^{2} .
\end{aligned}
$$

Substituting (38)-(41) into (37), we have

$$
\theta^{N} V(N) \leq \phi_{1}(\theta) \sup _{-\tau_{M} \leq s \leq 0}\|e(s)\|^{2}+\phi_{2}(\theta) \sum_{k=0}^{N} \theta^{k}\|e(k)\|^{2}
$$

where

$$
\begin{aligned}
\phi_{1}(\theta)= & \rho_{1}+\tau_{M}\left(\rho_{2}+\rho_{3}\right)+\rho_{4} \widehat{\mu}+\tau_{M} \theta^{\tau_{M}}(\theta-1) \\
& \times\left(\rho_{2}+\rho_{3}\right)+\rho_{4}(\theta-1) w(\theta) \\
\phi_{2}(\theta)= & (\theta-1) \rho_{1}-\theta \varepsilon+\tau_{M} \theta^{\tau_{M}}(\theta-1) \\
& \times\left(\rho_{2}+\rho_{3}\right)+\rho_{4}(\theta-1) w(\theta) .
\end{aligned}
$$

Because $\phi_{2}(1)<0$, it can be verified that there exists a scalar $\theta_{0}>1$ such that $\phi_{2}\left(\theta_{0}\right)<0$. Therefore, we can get that

$$
V(N) \leq \phi_{1}\left(\theta_{0}\right)\left(\frac{1}{\theta_{0}}\right)^{N} \sup _{-\tau_{M} \leq s \leq 0}\|e(s)\|^{2} .
$$

Meanwhile, it follows from (18) that $V(N) \geq \lambda_{\min }(P)\|e(N)\|^{2}$. Then, it can be obtained that

$$
\|e(N)\| \leq\left(\frac{\phi_{1}\left(\theta_{0}\right)}{\lambda_{\min }(P)}\right)^{1 / 2}\left(\frac{1}{\theta_{0}}\right)^{N / 2} \sup _{-\tau_{M} \leq s \leq 0}\|e(s)\| .
$$

According to Definition 5, we conclude that the system (9) is globally exponentially stable. This completes the proof.

Remark 7. In [30], the triple-integral term was first introduced in the Lyapunov functional since it could reduce the conservativeness of the stability criterion greatly. The tripleintegral term has been used to deal with the continuous-time neural networks, yet few present works have extended it to tackle the discrete-time neural networks. In this paper, a triple summation term is introduced in $V_{5}(k)$ which is helpful for the reduction of conservativeness.

Remark 8. In $[18,19]$, the authors concerned the discretetime fuzzy neural networks only with discrete-time delays. Meanwhile, the information on the considered time delays was not involved in the results. The condition given in Theorem 6 contains the time delay information. Thus the effect of the delays on the dynamical behavior of neural networks is considered in our result.

Remark 9. This work is the first attempt, to the best of our knowledge, to deal with the state estimation problem for discrete-time fuzzy cellular neural networks by using linear matrix inequality approach, not to mention the fact that the unbounded distributed delays are also involved. Compared with the results in the form of M-matrix in [18, 19], the result in the form of linear matrix inequalities (LMIs) is less conservative and can be easily solved by using the MATLAB LMI toolbox.

If we neglect the effect of the distributed delay term, the error system (9) reduces to

$$
\begin{aligned}
e(k+1)= & -(D+K C)(k)+A f(k) \\
& +B f(k-\tau(k))-K h(k) .
\end{aligned}
$$

For the system (46), we obtain the following result based on Theorem 6. 
Corollary 10. Suppose that assumption (H2) and condition (7) hold; the error system (46) is globally exponentially stable if there exist positive diagonal matrices $P, D_{1}$, and $D_{2}$, positive symmetric matrices $Q_{1}, Q_{2}, T$, and $R$, real matrix $N$, and scalars $\vartheta>0$ such that the following LMI holds:

$$
\Phi_{1}=\left[\begin{array}{cc}
\Psi_{1} & \xi_{1}^{T} P \\
* & -\Omega_{2}
\end{array}\right]<0,
$$

where

$$
\Psi_{1}=\left[\begin{array}{ccccccccc}
r_{1,1} & 0 & 0 & 0 & r_{1,5} & r_{1,6} & r_{1,7} & r_{1,8} & -N \\
* & -Q_{1} & 0 & 0 & 0 & 0 & 0 & 0 & 0 \\
* & * & -Q_{2} & 0 & 0 & 0 & 0 & 0 & 0 \\
* & * & * & r_{4,4} & 0 & 0 & 0 & L_{2} D_{2} & 0 \\
* & * & * & * & r_{5,5} & r_{5,6} & 0 & 0 & 0 \\
* & * & * & * & * & r_{6,6} & 0 & 0 & 0 \\
* & * & * & * & * & * & -D_{1} & 0 & 0 \\
* & * & * & * & * & * & * & -D_{2} & 0 \\
* & * & * & * & * & * & * & * & -9 I
\end{array}\right],
$$

with

$$
\begin{gathered}
r_{1,1}=-2 P D-N C-C^{T} N^{T}-2 P+Q_{1}+Q_{2} \\
+\left(\tau_{1}+1\right) T-\frac{2 \tau_{1}}{\tau_{2}+1} R-L_{1} D_{1}+\vartheta H^{T} H, \\
r_{1,5}=r_{1,6}=\frac{2}{\tau_{2}+1} R, \quad r_{1,7}=P A+L_{2} D_{1}, \\
r_{1,8}=P B, \quad r_{4,4}=-T-L_{1} D_{2}, \\
r_{5,5}=r_{5,6}=r_{6,6}=-\frac{2}{\tau_{1}\left(\tau_{2}+1\right)} R, \\
\quad \Omega_{2}=\frac{\tau_{1}}{2}\left(\tau_{2}+1\right) R-P, \\
\xi_{1}=[-(D+K C+I), 0,0,0,0,0, A, B,-K] .
\end{gathered}
$$

Moreover, the estimate gain matrix can be designed as $K=$ $P^{-1} N$.

Proof. Consider the following Lyapunov-Krasovskii functional:

$$
V(k)=\sum_{i=1}^{4} V(k),
$$

where

$$
\begin{aligned}
& V_{1}(k)=e^{T}(k) P e(k), \\
& V_{2}(k)=\sum_{i=k-\tau_{m}}^{k-1} e^{T}(i) Q_{1} e(i)+\sum_{i=k-\tau_{M}}^{k-1} e^{T}(i) Q_{2} e(i), \\
& V_{3}(k)=\sum_{i=-\tau_{M}+1}^{-\tau_{m}+1} \sum_{j=k-1+i}^{k-1} e(j)^{T} T e(j), \\
& V_{4}(k)=\sum_{i=-\tau_{M}}^{-\tau_{m}-1} \sum_{j=i l=k+j}^{-1} \sum^{k-1} \eta^{T}(l) R \eta(l) .
\end{aligned}
$$

Calculating the difference of $V(k)$ along the solution of system (46), we have

$$
\begin{aligned}
\Delta V_{1}(k)= & V_{1}(k+1)-V_{1}(k) \\
= & \eta^{T}(k) P \eta(k)+2 e^{T}(k) P \eta(k) \\
= & \eta^{T}(k) P \eta(k)+2 e^{T}(k) \\
& \quad \times P[-(D+K C+I) e(k)+A f(k) \\
& \quad+B f(k-\tau(k))-K h(k)] .
\end{aligned}
$$

$\Delta V_{2}(k), \Delta V_{3}(k)$, and $\Delta V_{4}(k)$ can be calculated using the same method in Theorem 6; hence the proof is omitted to avoid duplication. Then, we have

$$
\Delta V(k) \leq \delta_{1}^{T}(k)\left[\Psi_{1}+\xi_{1}^{T} \Omega_{1} \xi_{1}\right] \delta_{1}(k),
$$

where

$$
\begin{gathered}
\delta_{1}^{T}(k)=\left[e^{T}(k), e^{T}\left(k-\tau_{m}\right), e^{T}\left(k-\tau_{M}\right), e^{T}(k-\tau(k)),\right. \\
\sum_{i=k-\tau(k)}^{k-\tau_{m}-1} e^{T}(i), \sum_{i=k-\tau_{M}}^{k-\tau(k)-1} e^{T}(i), f^{T}(k), \\
\left.f^{T}(k-\tau(k)), h^{T}(k)\right] \\
\Omega_{1}=\frac{\tau_{1}}{2}\left(\tau_{2}+1\right) R+P .
\end{gathered}
$$

From $R>0, P>0$, and $\left(P-\Omega_{1}\right) \Omega_{1}^{-1}\left(P-\Omega_{1}\right) \geq 0$, we have

$$
-P \Omega_{1}^{-1} P \leq \Omega_{1}-2 P=\Omega_{2} .
$$

Noting $K=P^{-1} N$, it follows from (47) and (55) that

$$
\left[\begin{array}{cc}
\Psi_{1} & \xi_{1}^{T} P \\
* & -P \Omega_{1}^{-1} P
\end{array}\right]<0 .
$$

Pre- and postmultiplying (56), respectively, by $\operatorname{diag}\left\{I, \Omega_{1} P^{-1}\right\}$ we can obtain

$$
\left[\begin{array}{cc}
\Psi_{1} & \xi_{1}^{T} \Omega_{1} \\
* & -\Omega_{1}
\end{array}\right]<0 .
$$

By Schur complement, (57) implies that $\Psi_{1}+\xi_{1}^{T} \Omega_{1} \xi_{1}<0$. Thus there exists a constant $\varepsilon_{1}<0$ such that

$$
\Delta V(k) \leq \delta_{1}^{T}(k)\left[\Psi_{1}+\xi_{1}^{T} \Omega_{1} \xi_{1}\right] \delta_{1}(k) \leq \varepsilon_{1}\|e(k)\|^{2} .
$$

It follows from Lyapunov-Krasovskii stability theorem that the system (46) is globally asymptotically stable. The proof of the exponential stability of the system (46) is similar to Theorem 6 and hence it is omitted.

\section{Numerical Examples}

In this section, two numerical examples are introduced to illustrate the effectiveness of the proposed results. 
Example 1. Consider system (3) with the following parameters:

$$
\begin{gathered}
D=\left[\begin{array}{ccc}
0.6 & 0 & 0 \\
0 & 0.3 & 0 \\
0 & 0 & 0.8
\end{array}\right], \quad A=\left[\begin{array}{ccc}
-2.1 & 1.4 & 1.2 \\
0 & 1.6 & -2.3 \\
0 & 0.9 & 1.1
\end{array}\right], \\
B=\left[\begin{array}{ccc}
1.5 & 3.7 & -2.4 \\
1.3 & -1.5 & 1.4 \\
2.1 & 0 & -0.7
\end{array}\right], \quad V=\left[\begin{array}{ccc}
1.2 & -0.8 & -2.4 \\
-0.5 & 1.1 & 1.6 \\
3.1 & 1.7 & -2.1
\end{array}\right], \\
C=\left[\begin{array}{ccc}
1 & 0 & 0 \\
0 & 1 & 0
\end{array}\right], \quad U=\left[\begin{array}{l}
1 \\
1 \\
1
\end{array}\right], \quad I=\left[\begin{array}{l}
0.2 \\
0.3 \\
0.5
\end{array}\right], \\
\alpha=\beta=\left[\begin{array}{ccc}
0.1 & 0.05 & -0.2 \\
0.2 & -0.13 & -0.1 \\
-0.3 & 0.15 & 0.4
\end{array}\right], \quad X=Z=\left[\begin{array}{lll}
1 & 1 & 1 \\
0 & 1 & 1 \\
0 & 1 & 0
\end{array}\right] .
\end{gathered}
$$

The activation function and the nonlinear disturbance are taken as follows:

$$
\begin{gathered}
f(x)=\left(-\tanh \left(0.6 x_{1}\right), 0.4 \tanh \left(x_{2}\right), \tanh \left(0.8 x_{2}\right)\right)^{T}, \\
\varphi(k, x)=\left(0.2 \sin \left(x_{1}\right), 0.2 \sin \left(x_{2}\right), 0.2 \sin \left(x_{3}\right)\right)^{T} .
\end{gathered}
$$

It is easy to verify that

$$
\begin{gathered}
L_{1}=0, \quad L_{2}=\operatorname{diag}\{-0.3,0.2,0.4\}, \\
H=\left[\begin{array}{ccc}
0.2 & 0 & 0 \\
0 & 0.2 & 0
\end{array}\right]
\end{gathered}
$$

Choosing $\tau(t)=3.5+1.5 \sin (k \pi / 2), \mu_{m}=(2.4)^{-2 m}$. With the above parameters and by using the MATLAB LMI toolbox to solve the LMI in Theorem 6, we obtain the feasible solution as follows:

$$
\begin{gathered}
Q_{1}=\left[\begin{array}{ccc}
5.6538 & 0.3218 & -0.2439 \\
0.3218 & 7.5645 & 0.0879 \\
-0.2435 & 0.0879 & 0.2576
\end{array}\right], \\
Q_{2}=\left[\begin{array}{lll}
2.3359 & 0.1966 & 0.9296 \\
0.1966 & 4.3578 & 0.8385 \\
0.9296 & 0.8385 & 2.1764
\end{array}\right], \\
R=\left[\begin{array}{ccc}
8.6079 & 0.0148 & 0.5234 \\
0.0148 & 5.6131 & -1.6227 \\
0.5234 & -1.6227 & 3.2485
\end{array}\right], \\
S=\left[\begin{array}{ccc}
24.7993 & -2.4517 & 1.7653 \\
-2.4517 & 45.2584 & 4.3912 \\
1.7653 & 4.3912 & 18.2866
\end{array}\right],
\end{gathered}
$$

$$
\begin{gathered}
T=\left[\begin{array}{ccc}
4.1344 & 0.9635 & 1.1195 \\
0.1032 & 6.3798 & -2.3912 \\
1.1195 & -2.3912 & 11.4738
\end{array}\right] \\
N=\left[\begin{array}{cc}
9.3859 & 1.3260 \\
-0.9772 & 5.0316 \\
-0.1236 & 4.8976
\end{array}\right] \\
P=\operatorname{diag}\{7.6333,6.5716,4.4942\} \\
D_{1}=\operatorname{diag}\{17.2788,23.2664,9.4513\} \\
D_{2}=\operatorname{diag}\{8.1396,16.2168,7.2743\} \\
\vartheta=18.3817, \quad \varepsilon=13.6115
\end{gathered}
$$

Thus, the state estimator gain matrix can be designed as

$$
K=\left[\begin{array}{cc}
9.3859 & 1.3260 \\
-0.9772 & 5.0316 \\
-0.1236 & 4.8976
\end{array}\right]
$$

Therefore, it follows from Theorem 6 that the error system (9) is globally exponentially stable. The simulation results are shown in Figures 1 and 2 . In the simulation, the initial values $(0.3,-0.2,0.4)^{T}$ and $(0.1,0.2,0.2)$ are chosen for systems $(3)$ and (8), respectively.

Example 2. Consider system (46) with the following parameters:

$$
\begin{gathered}
D=\left[\begin{array}{ccc}
0.4 & 0 & 0 \\
0 & 0.3 & 0 \\
0 & 0 & 0.3
\end{array}\right], \quad A=\left[\begin{array}{ccc}
0.2 & -0.2 & 0.1 \\
0 & -0.3 & 0.2 \\
-0.2 & -0.1 & -0.2
\end{array}\right], \\
B=\left[\begin{array}{ccc}
-0.2 & 0.1 & 0 \\
-0.2 & 0.3 & 0.1 \\
0.1 & -0.2 & 0.3
\end{array}\right] .
\end{gathered}
$$

The activation function and the nonlinear disturbance are taken as follows:

$$
\begin{aligned}
& f(x)=0.25 \sin x+0.25 x \\
& \varphi(k, x)=0.2 \cos x+0.2 x
\end{aligned}
$$

It is easy to verify that

$$
L_{1}=0, \quad L_{2}=0.25 I, \quad H=0.4 I .
$$

Our purpose is to find the maximum allowable upper bound $\tau_{M}$ for different $\tau_{m}$ by solving the LMI (47) via the MATLAB LMI toolbox such that system (46) is globally exponentially stable. Now assume $\tau_{m}=4$; then by utilizing the result in [32] it is found that the maximum allowable upper bound $\tau_{M}$ is 9 . By using the method proposed in this paper, we find that the maximum allowable upper bound $\tau_{M}$ is 12. A more detailed comparison is given in Table 1 , from which we see that the stability criterion proposed in this paper is less conservative than the results proposed in $[21,32]$. 
TABLE 1: The maximum allowable upper bound $\tau_{M}$ for different $\tau_{m}$.

\begin{tabular}{lcccccc}
\hline Methods & $\tau_{m}=2$ & $\tau_{m}=3$ & $\tau_{m}=6$ & $\tau_{m}=11$ & $\tau_{m}=15$ & 19 \\
\hline [32, Theorem 1] & 7 & 8 & 11 & 16 & $\tau_{m}=18$ \\
Corollary 1 [21] & 7 & 9 & 12 & 17 & 21 & 23 \\
Theorem 6 & 10 & 11 & 14 & 19 & 22 \\
\hline
\end{tabular}
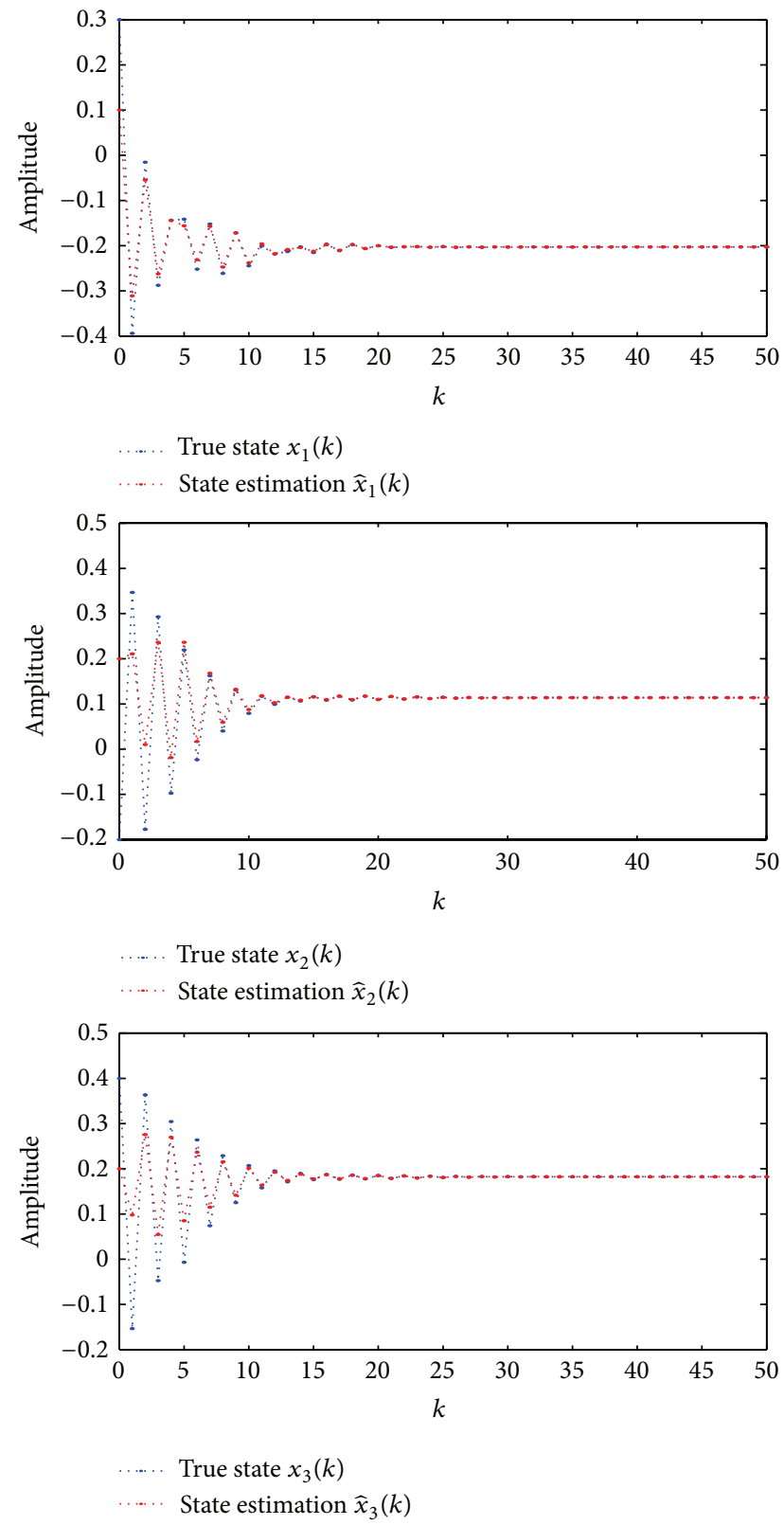

FIGURE 1: The responses of the true state $x(k)$ and the estimate state $\widehat{x}(k)$.

\section{Conclusion}

In this paper, we have studied the state estimation problem for a class of discrete-time fuzzy cellular neural networks with mixed time delays. A state estimator is designed to

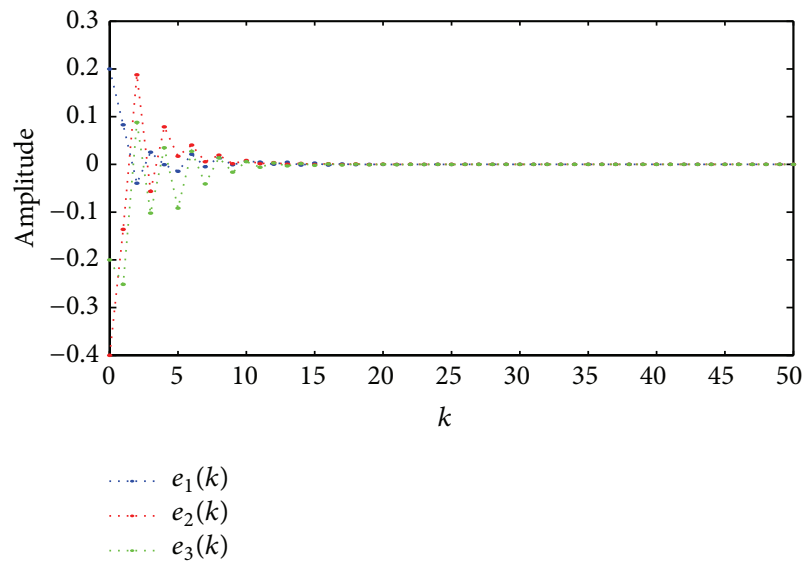

FIgURE 2: The responses of estimation error $e(k)$.

estimate the neuron states through available output measurements, such that the dynamics of the error-state system is exponentially asymptotically stable. By constructing a novel Lyapunov-Krasovskii functional containing a triple summation term and utilizing the LMI approach, some less conservative stability criteria in terms of LMI are derived to guarantee the existence of the asymptotic state estimator. It is worth noting that our developed approach can be effectively employed to investigate the state estimation and stability analysis problems for other discrete-time fuzzy delayed neural networks. Recently, a delay partition approach was proposed and has been verified to be very effective in reducing the conservatism of stability conditions. The delay partition approach also can be employed in our work to further reduce the conservatism. However, this approach will increase the computational complexity of the theorem because too many matrices were introduced. How to further reduce the conservatism by utilizing delay partition approach and, in the meantime, overcome the rapid increase in the computational complexity is still a challenging topic and will be investigated in our future work.

\section{Conflict of Interests}

The authors declare that there is no conflict of interests regarding the publication of this paper.

\section{Acknowledgments}

This work was supported by the National Natural Science Foundation of China (61305076) and by NNSF of China (11071254). 


\section{References}

[1] L. O. Chua and L. B. Yang, "Cellular neural networks: theory," IEEE Transactions on Circuits and Systems, vol. 35, no. 10, pp. 1257-1272, 1988.

[2] T. Yang, L.-B. Yang, C. W. Wu, and L. O. Chua, "Fuzzy cellular neural networks: theory," in Proceedings of the 4th IEEE International Workshop on Cellular Neural Networks, and Their Applications, pp. 181-186, June 1996.

[3] T. Yang, L.-B. Yang, C. W. Wu, and L. O. Chua, "Fuzzy cellular neural networks: applications," in Proceedings of the 4th IEEE International Workshop on Cellular Neural Networks, and Their Applications, pp. 225-230, June 1996.

[4] M. Syed Ali and P. Balasubramaniam, "Global asymptotic stability of stochastic fuzzy cellular neural networks with multiple discrete and distributed time-varying delays," Communications in Nonlinear Science and Numerical Simulation, vol. 16, no. 7, pp. 2907-2916, 2011.

[5] K. Yuan, J. Cao, and J. M. Deng, "Exponential stability and periodic solutions of fuzzy cellular neural networks with timevarying delays," Neurocomputing, vol. 69, no. 13-15, pp. 16191627, 2006.

[6] Z. W. Liu, H. G. Zhang, and Z. S. Wang, "Novel stability criterions of a new fuzzy cellular neural networks with timevarying delays," Neurocomputing, vol. 72, no. 4-6, pp. 1056-1064, 2009.

[7] X. D. Li, R. Rakkiyappan, and P. Balasubramaniam, "Existence and global stability analysis of equilibrium of fuzzy cellular neural networks with time delay in the leakage term under impulsive perturbations," Journal of the Franklin Institute, vol. 348, no. 2, pp. 135-155, 2011.

[8] X. M. Feng, F. Q. Zhang, and W. J. Wang, "Global exponential synchronization of delayed fuzzy cellular neural networks with impulsive effects," Chaos, Solitons and Fractals, vol. 44, no. 1-3, pp. 9-16, 2011.

[9] Q. T. Gan, R. Xu, and P. H. Yang, "Exponential synchronization of stochastic fuzzy cellular neural networks with time delay in the leakage term and reaction-diffusion," Communications in Nonlinear Science and Numerical Simulation, vol. 17, no. 4, pp. 1862-1870, 2012.

[10] Y. R. Liu, Z. D. Wang, and X. H. Liu, "Asymptotic stability for neural networks with mixed time-delays: the discrete-time case," Neural Networks, vol. 22, no. 1, pp. 67-74, 2009.

[11] H. W. Wang and Q. K. Song, "Synchronization for an array of coupled stochastic discrete-time neural networks with mixed delays," Neurocomputing, vol. 74, no. 10, pp. 1572-1584, 2011.

[12] H. Y. Li, C. Wang, P. Shi, and H. J. Gao, "New passivity results for uncertain discrete-time stochastic neural networks with mixed time delays," Neurocomputing, vol. 73, no. 16-18, pp. 3291-3299, 2010.

[13] T. Li, A. G. Song, and S. M. Fei, "Synchronization control for arrays of coupled discrete-time delayed Cohen-Grossberg neural networks," Neurocomputing, vol. 74, no. 1-3, pp. 197-204, 2010.

[14] M. J. Park, O. M. Kwon, S. M. Li, and E. J. Cha, "On synchronization criterion for coupled discrete-time neural networks with interval time-varying delays," Neurocomputing, vol. 99, no. 1, pp. 188-196, 2013.

[15] L. Tian, J. L. Liang, and J. D. Chao, "Robust observer for discrete-time Markovian jumping neural networks with mixed mode-dependent delays," Nonlinear Dynamics, vol. 67, no. 1, pp. 47-61, 2012.
[16] D. Zhang and L. Yu, "Passivity analysis for discrete-time switched neural networks with various activation functions and mixed time delays," Nonlinear Dynamics, vol. 67, no. 1, pp. 403411, 2012.

[17] Z. G. Wu, P. Shi, H. Y. Su, and J. Chu, "Dissipativity analysis for discrete-time stochastic neural networks with time-varying delays," IEEE Transactions on Neural Networks and Learning Systems, vol. 24, no. 3, pp. 345-355, 2013.

[18] Q. K. Song and J. D. Cao, "Dynamical behaviors of discretetime fuzzy cellular neural networks with variable delays and impulses," Journal of the Franklin Institute, vol. 345, no. 1, pp. 39-59, 2008.

[19] Y. K. Li and C. Wang, "Existence and global exponential stability of equilibrium for discrete-time fuzzy BAM neural networks with variable delays and impulses," Fuzzy Sets and Systems, vol. 217, pp. 62-79, 2013.

[20] Y. R. Liu, Z. D. Wang, and X. H. Liu, "State estimation for discrete-time Markovian jumping neural networks with mixed mode-dependent delays," Physics Letters A: General, Atomic and Solid State Physics, vol. 372, no. 48, pp. 7147-7155, 2008.

[21] H. B. Bao and J. D. Cao, "Delay-distribution-dependent state estimation for discrete-time stochastic neural networks with random delay," Neural Networks, vol. 24, no. 1, pp. 19-28, 2011.

[22] S. Lakshmanan, J. H. Park, H. Y. Jung, and P. Balasubramaniam, "Design of state estimator for neural networks with leakage, discrete and distributed delays," Applied Mathematics and Computation, vol. 218, no. 22, pp. 11297-11310, 2012.

[23] Z. Wang, Y. Liu, X. Liu, and Y. Shi, "Robust state estimation for discrete-time stochastic neural networks with probabilistic measurement delays," Neurocomputing, vol. 74, no. 1-3, pp. 256264, 2010.

[24] Z. G. Wu, H. Y. Su, and J. Chu, "State estimation for discrete Markovian jumping neural networks with time delay," Neurocomputing, vol. 73, no. 10-12, pp. 2247-2254, 2010.

[25] D. Zhang and L. Yu, "Exponential state estimation for Markovian jumping neural networks with time-varying discrete and distributed delays," Neural Networks, vol. 35, no. 11, pp. 103-111, 2012.

[26] D. Zhang, L. Yu, Q. G. Wang, and C. J. Ong, "Estimator design for discrete-time switched neural networks with asynchronous switching and time-varying delay," IEEE Transactions on Neural Networks and Learning Systems, vol. 23, no. 5, pp. 827-834, 2012.

[27] P. Balasubramaniam, M. Kalpana, and R. Rakkiyappan, "State estimation for fuzzy cellular neural networks with time delay in the leakage term, discrete and unbounded distributed delays," Computers and Mathematics with Applications, vol. 62, no. 10, pp. 3959-3972, 2011.

[28] Y. R. Liu, Z. D. Wang, and X. H. Liu, "Global exponential stability of generalized recurrent neural networks with discrete and distributed delays," Neural Networks, vol. 19, no. 5, pp. 667-675, 2006.

[29] Y. R. Liu, Z. D. Wang, J. L. Liang, and X. H. Liu, "Synchronization and state estimation for discrete-time complex networks with distributed delays," IEEE Transactions on Systems, Man, and Cybernetics, B: Cybernetics, vol. 38, no. 5, pp. 1314-1325, 2008.

[30] J. Sun, G. P. Liu, and J. Chen, "Delay-dependent stability and stabilization of neutral time-delay systems," International Journal of Robust and Nonlinear Control, vol. 19, no. 10, pp. 13641375, 2009.

[31] Q. K. Song and Z. D. Wang, "A delay-dependent LMI approach to dynamics analysis of discrete-time recurrent neural networks 
with time-varying delays," Physics Letters A: General, Atomic and Solid State Physics, vol. 368, no. 1-2, pp. 134-145, 2007.

[32] S. S. Mou, H. J. Gao, W. Y. Qiang, and Z. Y. Fei, "State estimation for discrete-time neural networks with time-varying delays," Neurocomputing, vol. 72, no. 1-3, pp. 643-647, 2008. 


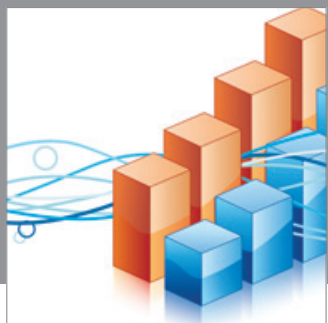

Advances in

Operations Research

mansans

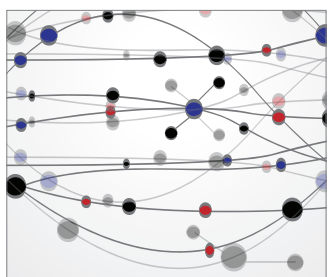

The Scientific World Journal
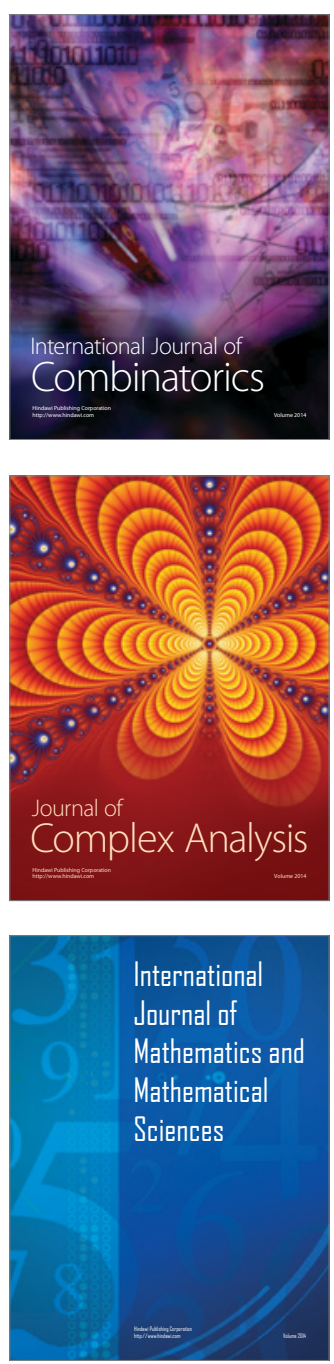
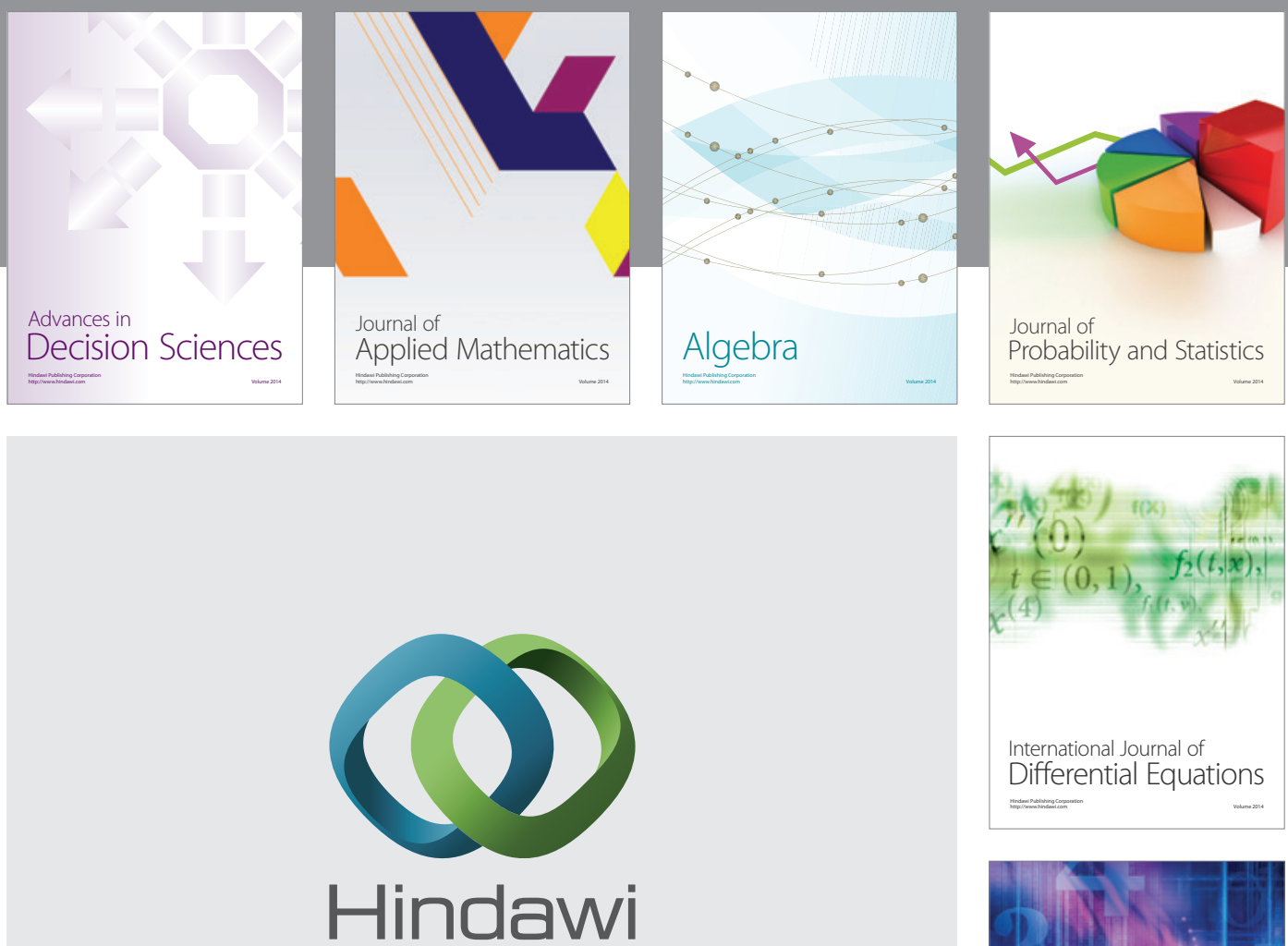

Submit your manuscripts at http://www.hindawi.com
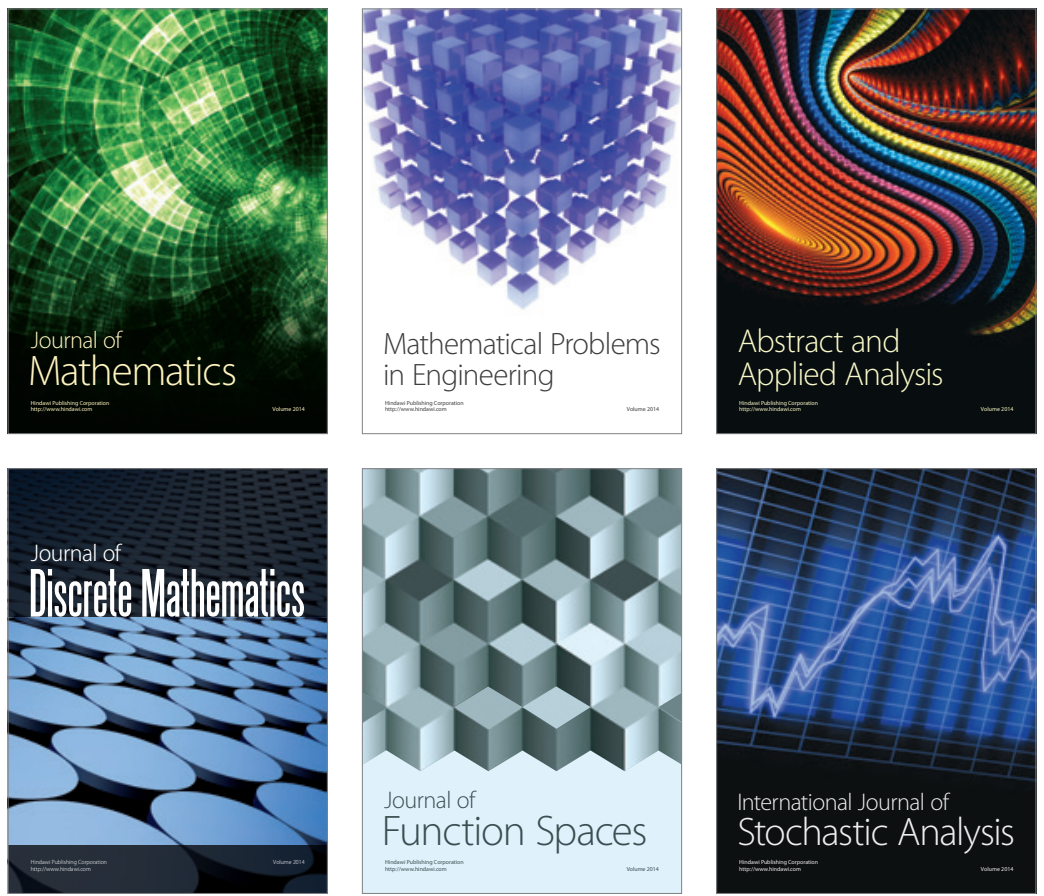

Journal of

Function Spaces

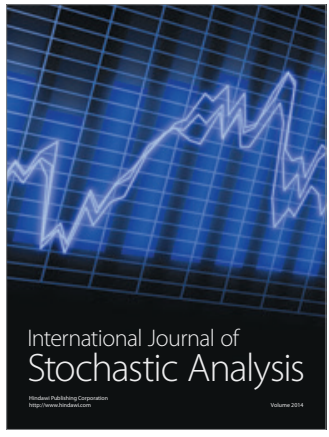

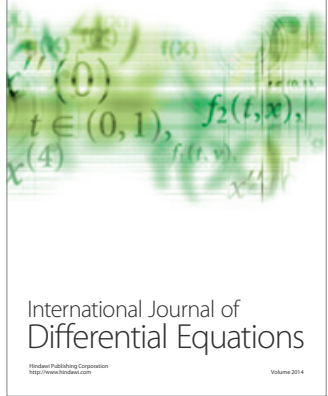
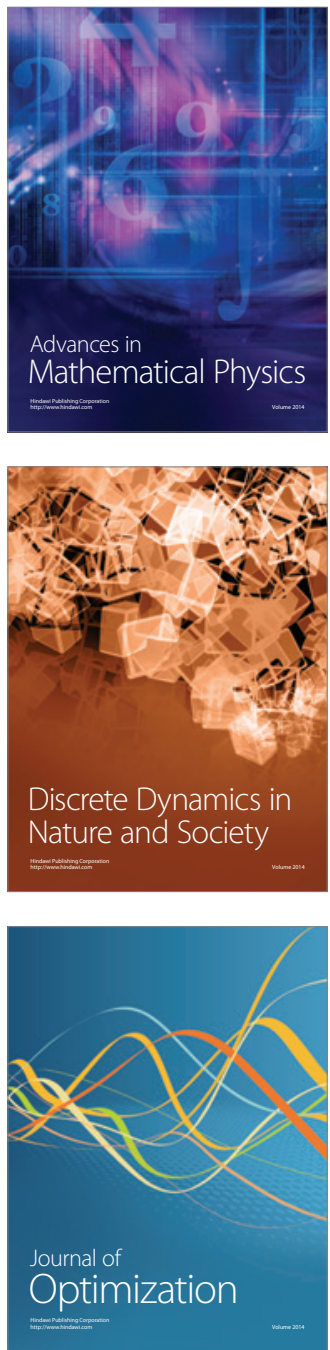